\title{
Transmutation of LLFP by Irradiation of Neutrons on Muon Catalyzed Fusion (MCF) Reactor
}

\author{
Norimasa YAMAMOTO, Motoyasu SATO, Hirohisa TAKANO and Atsuo IIYOSHI \\ Chubu University, 1200 Matsumoto-cho, Kasugai-city, Aichi 487-8501, Japan
}

(Received 25 August 2020 / Accepted 14 April 2021)

\begin{abstract}
The objective of this article is to provide a database for the transmutation of LLFP (long-lived fission products) using neutrons of muon-catalyzed nuclear fusion (MCF). As examples of LLFP with a natural half-life of more than $10^{5}$ years, four nuclides, ${ }^{107} \mathrm{Pd},{ }^{135} \mathrm{Cs}$, ${ }^{79} \mathrm{Se}$ and ${ }^{93} \mathrm{Zr}$, are chosen. Taking simplified geometrical models of the neutron source and blanket, which appear in the conceptual design of in-flight MCF, the nuclide production yield was calculated by a three-dimensional Monte Carlo calculation based on nuclear data. The number of neutrons and flux, which are necessary to convert half of the initial LLFP amount into a stable nucleus, are obtained. We also investigated the method of controlling two competing reactions of the nuclear fractions by fast neutrons, called the $(n, 2 n)$ reaction, and the neutron captures of the thermal neutrons. Theoretical simulation studies have revealed the quantity of LLFP that is detoxified by transmutation under the condition that the fusion neutrons are continuously irradiated to LLFP for approximately 10 years with a flux of $10^{19} \mathrm{~m}^{-2} \mathrm{~s}^{-1}$.
\end{abstract}

(C) 2021 The Japan Society of Plasma Science and Nuclear Fusion Research

Keywords: muon-catalyzed nuclear fusion, LLFP, neutron, transmutation by neutron

DOI: $10.1585 /$ pfr. 16.1405074

\section{Introduction}

Controlled thermonuclear fusion research has been developed worldwide and has reached the stage of the construction of the ITER (International Thermonuclear Experimental Reactor), in which experiments of deuteriumtritium (D-T) fusion reactions are planned [1]. Alpha particles and $14.1 \mathrm{MeV}$ neutrons are generated in the D-T reaction. These neutrons have also attracted attention to reduce radioactive waste LLFP (long-lived fission products) because of their high and nearly monochromatic energy spectrum $[2,3]$.

Transmutation by neutrons is roughly divided into two processes. For example, when a nucleus of radioactive ${ }^{93} \mathrm{Zr}$ with a half-life of approximately $10^{6}$ years collides with fast neutrons, it can be transmuted into stable ${ }^{92} \mathrm{Zr}\left({ }^{93} \mathrm{Zr}+\right.$ $\mathrm{n}(14.1 \mathrm{MeV}) \rightarrow{ }^{92} \mathrm{Zr}($ stable $\left.)+2 \mathrm{n}\right)$. However, by colliding with thermal neutrons, the nucleus captures neutrons and is transmuted into stable ${ }^{94} \mathrm{Zr}\left({ }^{93} \mathrm{Zr}+\mathrm{n} \rightarrow{ }^{94} \mathrm{Zr}\right.$ (stable)). Neutrons from D-T nuclear fusion are thought to have high monochromatic energy, which is advantageous for the treatment of radioactive waste in the first path of these two examples. Thus, the use of neutrons from fusion reactors for the processing of LLFP has been discussed in the literature, e.g., $[4,5]$.

Various approaches for making fusion-generated neutrons have been studied, in addition to the magnetic confinement of high-energy plasmas. In particular, muoncatalyzed fusion (MCF) has been studied for more than half a century [6]. In this approach, muons play a role to

author'se-mail: yamamoto-no@isc.chubu.ac.jp reduce the Coulomb repulsion force between reacting $\mathrm{D} / \mathrm{T}$ nuclei (so as to enhance the fusion cross-section), by forming a muonic atom, in which the electron is replaced by negative muon [7]. Once the fusion reaction takes place, the muons are released (and then are effective in the following fusion reaction) so that they play the role of a 'catalyst' in accelerating the fusion reaction. This catalytic work is effective during the lifetime of a muon $(\sim 2.2 \mathrm{mi}-$ croseconds). The cycle number, i.e., the number of fusion reactions that one muon induces during its lifetime, is the key for MCF. Near the end of the last century, this cycle number reached approximately 150 , which was approximately half for energy break-even at that time but might be high enough for the neutron source to treat LLFP [6]. The issue of MCF for further application (in the last century) was that MCF was experimentally analyzed by use of solid hydrogen so that the extension to practical use was limited.

Recently, two new ideas have been developed, which substantially enhance the advantages of MCF. The first is the concept of in-flight-MCF $[8,9]$. It has been shown that the cross-section of MCF can be dramatically enhanced if the relative energy between the muonic tritium atom and deuterium nucleus reaches the range of $\mathrm{keV}$ [10]. The second concept is the idea of using high-density shock formation in gaseous $\mathrm{D} / \mathrm{T}$ to replace the $\mathrm{D} / \mathrm{T}$ ice to sustain the high-density target medium where MCF takes place [11]. If this type of Dual Muon Catalyzed Fusion (DMCF) reactor can be designed, the neutron is generated in a compact area (possibly a spheroidal shape with a small diameter of a few $\mathrm{cm}$ ); that is, good access with a wide solid angle is available. 
This article describes the transmutation reduction of LLFP by neutrons from a local source of D-T fusion reactions. The transmutation efficiencies of various LLFPs $\left({ }^{107} \mathrm{Pd},{ }^{135} \mathrm{Cs},{ }^{79} \mathrm{Se}\right.$, and $\left.{ }^{93} \mathrm{Zr}\right)$ are calculated using a model in which the neutron source is centered on a small sphere of a single nuclide. The calculation considers the different types of blankets that surround the neutron source. The impacts of sizes and structures (component, thickness, etc.) are analyzed on the efficiency of transmutation for LLFP elements.

\section{Calculations}

This section describes how to calculate the yield of nuclides produced from LLFP by D-T fusion neutrons. The effective half-life of number of nuclides to the initially loaded LLFP is used as a measure of progress of the transmutation. To estimate the rate of reaction, the process must be known in which the incident neutrons collide with the LLFP and the transmuted nuclides. The progress of this collision process is calculated by applying the 3D Monte Carlo code PHITS (ver. 2.82) [12]. This code uses the nuclear database JENDL-4.0 [13] (elastic scattering, inelastic scattering (n, 2n) reactions, neutron capture, etc.).

\subsection{Model for calculation}

In the calculation of transmutation of LLFP by fusion neutrons using PHITS, the initially loaded LLFP was positioned in a spherical shell, and a neutron source with mono energy of $14.1 \mathrm{MeV}$ was positioned in the shell center, as shown in Fig. 1 (a). The inner diameter of the spherical shell was $0.5 \mathrm{~m}$. Therefore, the number of $10^{18} \mathrm{~s}^{-1}$ neutrons corresponded to a neutron flux of approximately $10^{18} \mathrm{~m}^{-2} \mathrm{~s}^{-1}$ (interior surface area is $0.785 \mathrm{~m}^{2}$ ). When the thickness of the LLFP was thin, neutrons easily passed through the LLFP. When thick, various processes competed. Since the degree of competition of various processes strongly depends on the thickness of the initially loaded LLFP, this paper mainly shows the result of the thickness dependence. (a)

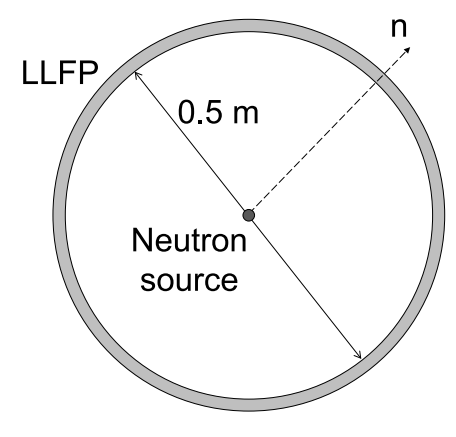

(b)
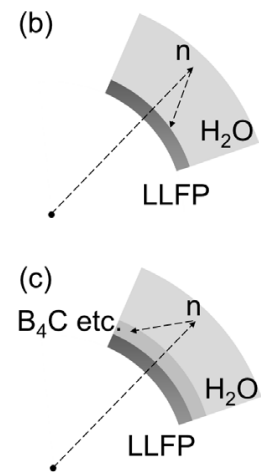

Fig. 1 Geometry and nuclear characteristics on simulation by the PHITS code.
Figure 1 (b) shows the geometry when light water is positioned as a moderator and coolant on the outside of the initial loaded LLFP. The results are shown in Fig. 4. Figure 1 (c) shows the geometry of the case where a substance is sandwiched between LLFP and light water, and the result is shown in Fig. 5.

\subsection{Effective half-life}

The effective half-life is an important measure for transmutation of LLFP. For single target nuclide, time dependence of the number of target nuclide $N(t)$ by irradiation of neutrons is as follows:

$$
\frac{d N}{d t}=-\varphi \sigma N
$$

where $\varphi$ is the neutron flux, and $\sigma$ is the effective cross section by neutron capture and (n, $2 n$ ) reaction. For LLFP, the decay constant of radioactive decay is negligible. The solution of the differential Eq. (1) is as follows:

$$
N(t)=N_{0} \exp (-\varphi \sigma t)
$$

where $N_{0}$ is the number of nuclides in the initially loaded LLFP. The time that $N_{0}$ is halved, the effective half-life, $T_{1 / 2}$, are written as follows:

$$
T_{1 / 2}=\frac{\ln 2}{\varphi \sigma} .
$$

If the factor $\varphi \sigma$ is known, the half-life time can be cleared. PHITS simulations estimate the nuclide production yield, $h$, per neutron as follows:

$$
h=\frac{\varphi}{n} \sigma N_{0}
$$

where $n$ is the number of neutrons based on the neutron flux $\varphi$. Substituting Eq. (4) into Eq. (3), the effective halflife $T_{1 / 2}$ is denoted by production yield $h$ :

$$
T_{1 / 2}=\ln 2 \frac{N_{0}}{n h}
$$

\subsection{Geometry of production yields in the shell}

The production yields $h$ contains of reactions of $(\mathrm{n}, 2 \mathrm{n})$ nuclear fragmentations and neutron capture that are investigated in the spherical model depending on Eq. (1) Eq. (5) by varying the thickness of the shell made by ${ }^{107} \mathrm{Pd},{ }^{135} \mathrm{Cs}{ }^{79} \mathrm{Se}$ and ${ }^{93} \mathrm{Zr}$.

The neutrons of $14.1 \mathrm{MeV}$ are generated in D-T fusion. In the four nuclides, threshold energy of cross section of the (n, $2 n$ ) reaction by neutron collision is 6 to $8 \mathrm{MeV}$, so that LLFP is transmuted by $(n, 2 n)$ reaction by D-T fusion neutrons. When the target is thick, neutrons of 80 to $90 \%$ cause $(n, 2 n)$ reactions, and the remaining neutrons slow down by repeating elastic and inelastic scattering. The neutrons radiated by the $(n, 2 n)$ reaction contribute to the transmutation of neutron capture. 


\section{Results}

\subsection{Transmutation with (n, 2n) nuclear frag- mentations}

Figure 2 shows the nuclide production yield $h$ for each LLFP, (a) ${ }^{107} \mathrm{Pd}$, (b) ${ }^{135} \mathrm{Cs}$, (c) ${ }^{79} \mathrm{Se}$, and (d) ${ }^{93} \mathrm{Zr}$ versus the thickness of the spherical shells. The dotted, dashed and solid lines correspond to $(n, 2 n)$ reactions, neutron captures and the sum of the two, respectively. By varying the thickness from $5 \mathrm{~mm}$ to $5 \mathrm{~m}$, the optimum thicknesses are found to be $0.1 \mathrm{~m}$. The nuclide production yields from the $(n, 2 n)$ reaction are equal to those from neutron capture, as indicated in Fig. 2; they are $4 \mathrm{~m}$ for ${ }^{107} \mathrm{Pd}, 0.3 \mathrm{~m}$ for ${ }^{135} \mathrm{Cs}$ and $0.4 \mathrm{~m}$ for ${ }^{79} \mathrm{Se}$ and ${ }^{93} \mathrm{Zr}$. If the target thickness is thinner than these values, the $(n, 2 n)$ reaction is the dominant mechanism. If the thickness of the LLFP target is thin, a part of the high-energy neutrons undergoes a (n, 2n) reaction. If the thickness of the LLFP target is too thin, a part of the high-energy neutrons penetrates the target without making a (n, 2n) reaction. However, Therefore, with a sufficiently thick target, the nuclide production yield exceeds

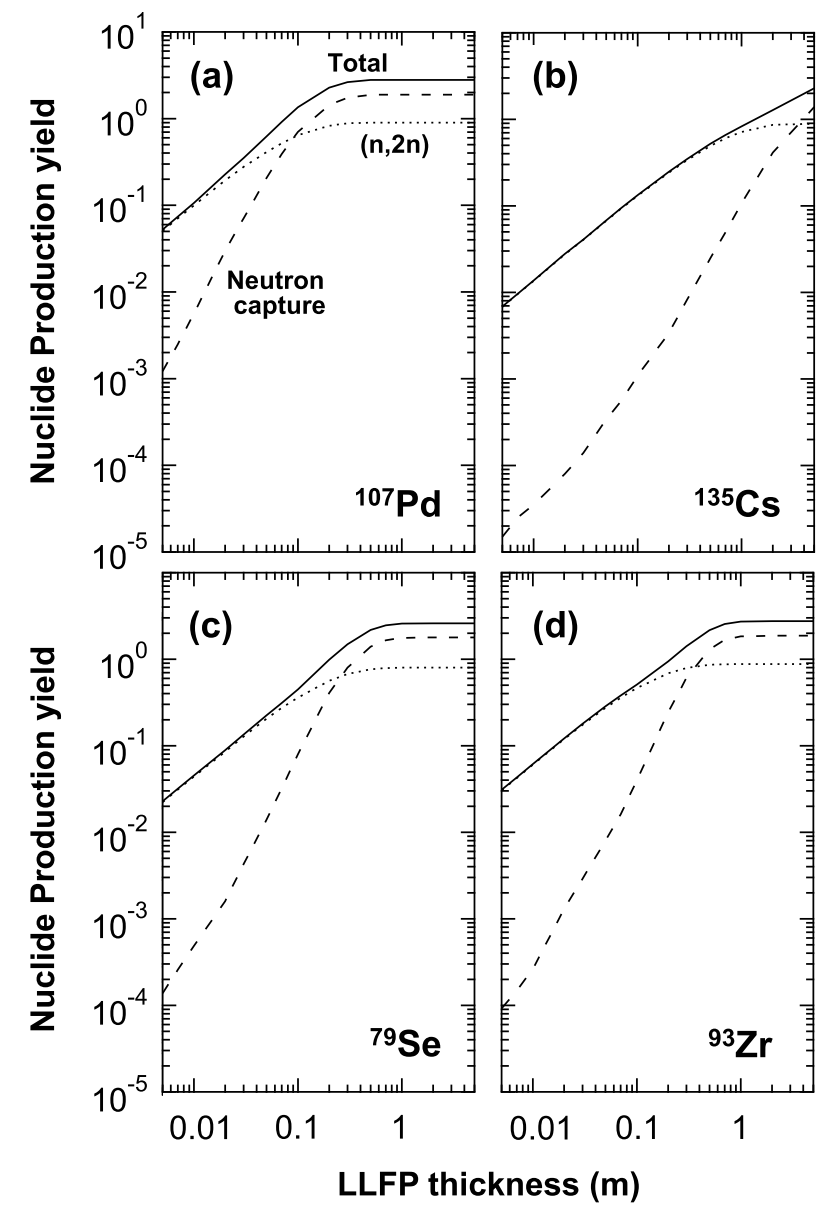

Fig. 2 Nuclide production yields for each LLFP thickness for a neutron with energy of $14.1 \mathrm{MeV}$ (DT). Dotted, dashed and solid lines are the nuclide production yield by the (n, $2 n$ ) reaction, neutron capture and sum of these, respectively. (a) ${ }^{107} \mathrm{Pd}$, (b) ${ }^{135} \mathrm{Cs}$, (c) ${ }^{79} \mathrm{Se}$, and (d) ${ }^{93} \mathrm{Zr}$. unity, reaching approximately 2.7. Transmutation by neutrons with an energy of $2.45 \mathrm{MeV}$ (D-D fusion) is caused only by the neutron capture reaction, and the yield cannot exceed unity by maximum.

\subsection{Doses of the neutron radiation}

Figure 3 shows the neutron irradiation periods required for transmutation, defined by Eq. (5). The neutrons of $10^{17}, 10^{18}$ and $10^{19} \mathrm{~s}^{-1}$ correspond to fluxes of approximately $10^{17}, 10^{18}$ and $10^{19} \mathrm{~m}^{-2} \mathrm{~s}^{-1}$, respectively. The results of $T_{1 / 2}$ on the four nuclides demonstrate the same tendency. This is because there is no large difference in the cross-section of the $(n, 2 n)$ reaction for an incident energy of $14.1 \mathrm{MeV}$. The slight difference between the four nuclides reflects the difference in the mass number density of the four targets; for example, palladium is the heaviest at approximately $11 \times 10^{3} \mathrm{~kg} / \mathrm{m}^{3}$, and cesium is the lightest at approximately $1.9 \times 10^{3} \mathrm{~kg} / \mathrm{m}^{3}$. When the target thickness is $100 \mathrm{~mm}$ or less, the irradiation time is almost constant, but when it exceeds $100 \mathrm{~mm}$, the necessary irradiation time increases sharply. When the LLFP $100 \mathrm{~mm}$ thickness is converted into weight, it corresponds to approximately $1200 \mathrm{~kg}, 200 \mathrm{~kg}, 500 \mathrm{~kg}$ and $700 \mathrm{~kg}$ for ${ }^{107} \mathrm{Pd}$, ${ }^{135} \mathrm{Cs},{ }^{79} \mathrm{Se}$ and ${ }^{93} \mathrm{Zr}$, respectively. From here, we will discuss the cases that include light water, so we will not mention cesium.

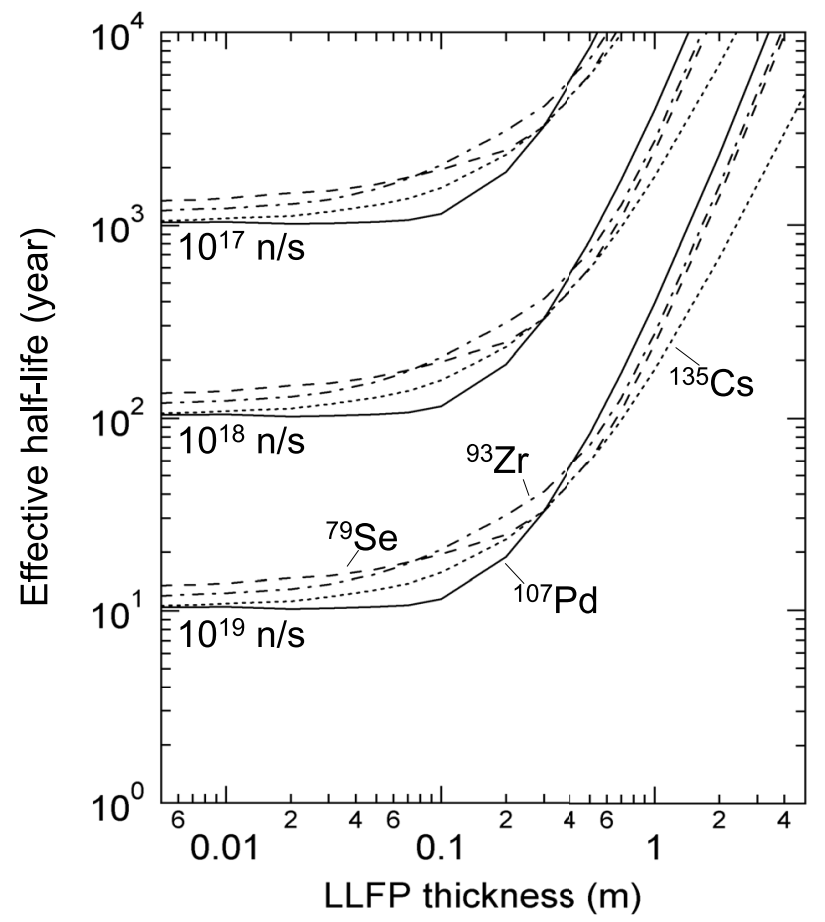

Fig. 3 Neutron irradiation time $T_{1 / 2}$ and neutron dose required $\left(10^{17}, 10^{18}\right.$, and $\left.10^{19} \mathrm{~s}^{-1}\right)$ to transmute half of the initial LLFP amount for each LLFP thickness. The neutron energy is $14.1 \mathrm{MeV}$. 


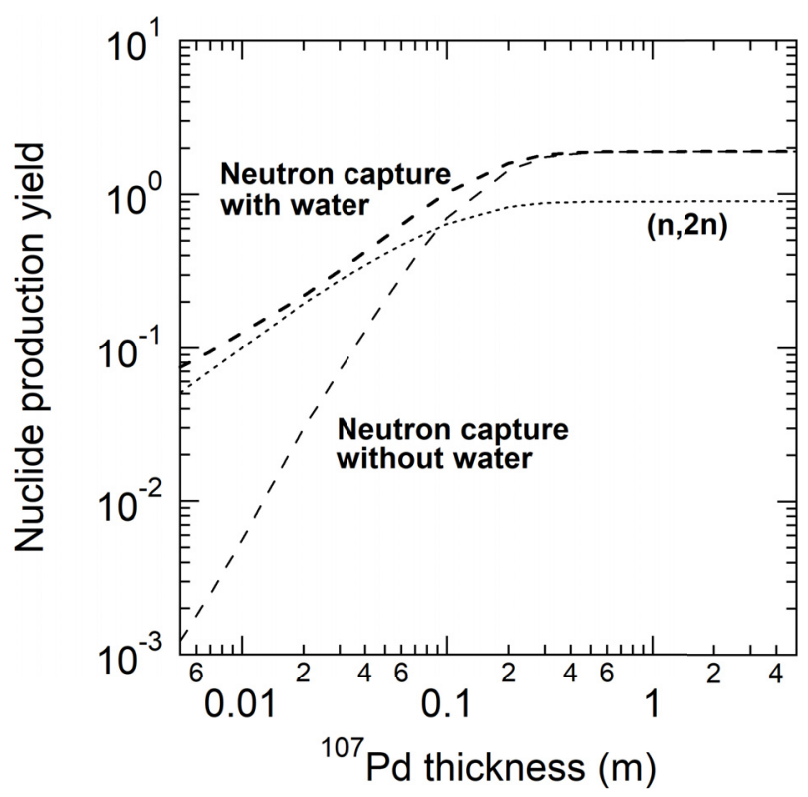

Fig. 4 Nuclide production yield with and without water outside the LLFP (see Fig. 1 (b)). The thin and thick dashed lines indicate the yield with water and without water, respectively. The dotted line is the yield by the (n, $2 n)$ reaction.

\subsection{Layout of neutron source, LLFP and cooling/shielding materials}

It should be noted that the outside of the sphere is the free space in the calculation. In practice, neutrons must be absorbed and sealed by materials that are water, metal materials, concrete, etc. on the outside surface of the reactor wall, which act as moderators and cooling solvents. Neutrons leaving the LLFP target are scattered by moderators, and after sufficient deceleration, they are incident again on the LLFP target, and transmutation can occur by neutron capture reaction. This is noteworthy when the thickness of the LLFP target is thin. Figure 4 shows the nuclide production yield when light water $\left(\mathrm{H}_{2} \mathrm{O}\right)$ with a thickness of $0.3 \mathrm{~m}$ is positioned outside the target LLFP (see Fig. 1 (b)). The results in Fig. 4 show that when water is positioned outside the LLFP, the nuclide production yield of neutron capture greatly increases. ${ }^{107} \mathrm{Pd},{ }^{79} \mathrm{Se}$, and ${ }^{93} \mathrm{Zr}$ become stable nuclides in both transmutation by the $(n, 2 n)$ reaction and transmutation by neutron capture.

\subsection{Cooling method for LLFP and Sub- stances}

The geometric calculation of Fig. 5 is shown in Fig. 1 (c). Metals and materials including boron of $\mathrm{W}$ [14], $\mathrm{Fe}, \mathrm{Mg}\left(\mathrm{BH}_{4}\right)_{2}$, and $\mathrm{B}_{4} \mathrm{C}$ as a suppression material of low energy neutron reentry were researched. Tungsten is used as the material for the fusion reactor, and boron is known as a substance that absorbs neutrons. The LLFP target is ${ }^{107} \mathrm{Pd}$ with a thickness of $100 \mathrm{~mm}$. The nuclide production yield by neutron capture when the outside of the LLFP tar-

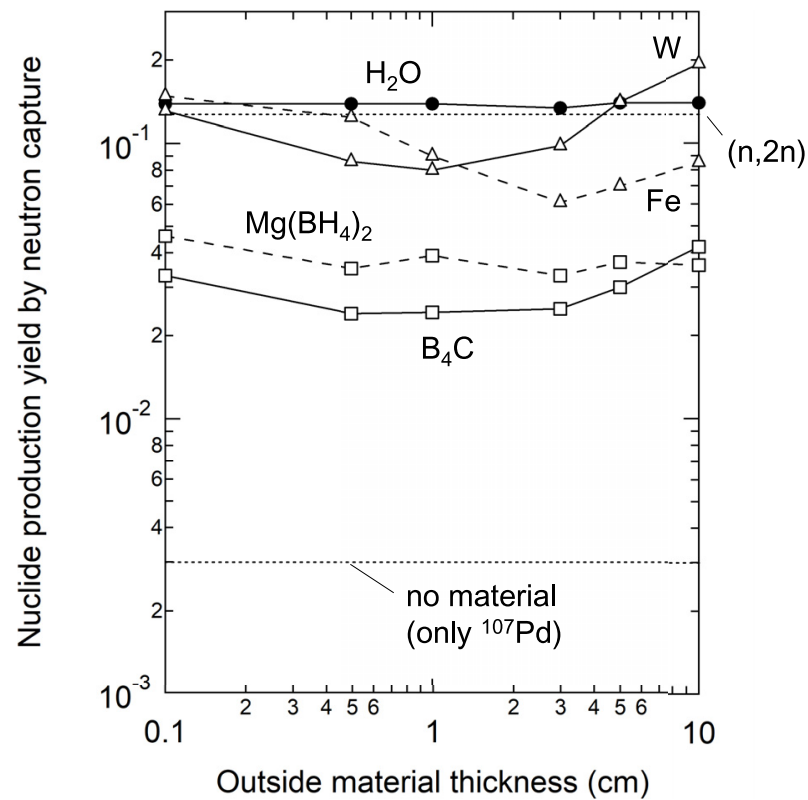

Fig. 5 Nuclide production yield due to neutron capture on various neutron re-incident suppression materials (See Fig. 1 (c)). The structure is LLFP-neutron re-incident suppression material-light water. The LLFP target is ${ }^{107} \mathrm{Pd}$.

get is only light water is almost equal to the yield by the $(\mathrm{n}, 2 \mathrm{n})$ reaction. In the case of tungsten $(\mathrm{W})$ or iron $(\mathrm{Fe})$ positioned between light water and LLFP, the yield by neutron capture is slightly reduced more than that without the materials.

Then, it was found that the material containing boron, which is said to have a neutron absorbing effect, suppresses the yield by neutron capture to approximately $1 / 5$ or less at a thickness of approximately $5-30 \mathrm{~mm}$. There is a possibility to be transmutation by a predominantly $(n, 2 n)$ reaction using $14.1 \mathrm{MeV}$ neutrons by disposing of $\mathrm{B}_{4} \mathrm{C}$ with approximately $5-30 \mathrm{~mm}$ between the light water and LLFP.

The energy of neutrons at $14.1 \mathrm{MeV}$ and $10^{19} \mathrm{~s}^{-1}$ is 22.56 MW in terms of the heat quantity. Almost all of this energy is consumed for heating LLFP, substrates and light water, as shown in the previous section. To keep the materials stable, they will eventually be water-cooled. The maximum thickness of the LLFP is $100 \mathrm{~mm}$. The heat that accompanies the $(n, 2 n)$ reaction that occurs inside the LLFP conducts heat inside the LLFP and is dissipated at the contact surface with the water behind it. The substrate is the part that absorbs the most neutron energy. A large surface area is required to cool the surface with water. In an actual neutron irradiation furnace, instead of $\mathrm{B}_{4} \mathrm{C}$ (which is a ceramic), a structure of a water tube boiler in which boron water flows at a high speed is recommended. This boron water is led to another secondary cooler and finally converted into electric power. 


\section{An Example of Neuron Source by Muon Catalyzed Fusion Reactor}

The discussions in this article were performed with an idealized geometry for the neutron source. As the design study for the DMCF reactor will be more realistic geometries of the neutron source and blanket design shall be considered. Figure 6 shows an example of the cross-sectional view of the fusion core zone of DMCF reactors.

The merit of muon catalyzed fusion is that "No particle confinement nor Energy confinement system is required". Because it satisfies the geometric condition to be a point source located in the center of the sphere. The geometry of such point sources is topologically impossible in magnetic fusion on a confinement along the magnetic line of force.

For example, in Fig. 6 geometry, the diffusive length of muon atom is limited in $1 \mathrm{~cm}^{3}$ area. For example, at a density of $10^{22} \mathrm{~cm}^{-3}(50 \mathrm{MPa})$, the diffusion region is coordinated to $1 \mathrm{~cm}$ radius spheric. It can be treated as a point neutron source and neutrons spread into $4 \pi$ direction almost uniformly from the center. The example will produce neutrons of $10^{19} \mathrm{~s}^{-2}$ and $28 \mathrm{MW}$ thermal power continuously.

The reaction zone can be hold by fluid dynamic force. As illustrated in Fig. 6, installing a pair of sharp wedges in the supersonic flow, the symmetric oblique shocks are excited and it goes finally to stagnation so called Mach shock where the density increased to $>10^{22} \mathrm{~cm}^{-3}$. The fresh muons are injected to the high density area and generates muon catalyzed fusion cycles in it. The diffusive force balances with the dynamic pressure to the Mach shock front. The highspeed hydrogen gas flow cools the reactor wall limiting maximum temperature in the fusion core to less than $500 \mathrm{~K}$.

The ram jet structures are exposed to the high flux of neutrons with 14.1 MeV. A major engineering issue is "selection of materials that can be withstand to the high-dose $10^{19} \mathrm{~s}^{-1}$ of fast neutrons". Application of LLFP might be the best choice for the first wall. The activation process of

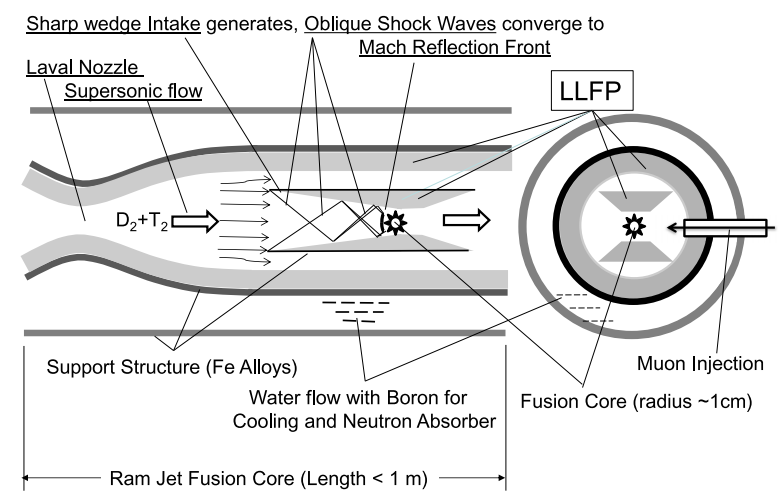

Fig. 6 The concept of muon catalyzed Fusion core held by ramjet shock structure.
Zirconium $(\mathrm{Zr})$ to neutron irradiation is shown in the previous section. The ${ }^{92} \mathrm{Zr}$ is converted to ${ }^{93} \mathrm{Zr}$, which is a radiative long-lived fission product (LLFP). However, ${ }^{93} \mathrm{Zr}$ gets transmutation to the stable end of ${ }^{94} \mathrm{Zr}$. The 10 - 30 years of irradiation to the $100 \%{ }^{93} \mathrm{Zr}$ transmute the $50 \%$ of it to the stable isotopes. The steel structure supports the LLFP layers from the back side where the circulating Bolon water and outer stainless-steel jacket covered absorbs all the reduced neutron energy.

\section{Summary and Conclusions}

In this article, the neutron irradiation time is evaluated as 10 - 30 years at $10^{19} \mathrm{~s}^{-1}$ of $14.1 \mathrm{MeV}$ neutrons for the LLFP in the test blanket $(100 \mathrm{~mm}$ in thickness: if converted into weight, this corresponds to approximately $1200 \mathrm{~kg}$, $200 \mathrm{~kg}, 500 \mathrm{~kg}$ and $700 \mathrm{~kg}$ for ${ }^{107} \mathrm{Pd},{ }^{135} \mathrm{Cs},{ }^{79} \mathrm{Se}$ and ${ }^{93} \mathrm{Zr}$, respectively). If one employs the case of a neutron generation amount of $10^{18} \mathrm{~s}^{-1}$, the irradiation time exceeds 100 years. Therefore, the high-energy neutrons of $14.1 \mathrm{MeV}$ with a magnitude of $10^{19} \mathrm{~s}^{-1}\left(=10^{19} \mathrm{~m}^{-2} \mathrm{~s}^{-1}\right)$ is desirable, considering the service life, etc., of the processing equipment. This research revealed that nuclear fusion neutrons from the presently designed in-flight MCF reactor can be of practical use for transforming LLFP.

If one interprets the outcome of this analysis conversely, the nuclear transmutation of LLFP, proposed by this research, is applied to the first wall cover materials. The use of LLFP could provide a valuable possibility for expanding the capability of conventional nuclear fusion reactors.

\section{Acknowledgments}

This work was funded by the ImPACT Program of Council for Science, Technology and Innovation (Cabinet Office, Government of Japan). This work was partially supported by a grant for research by Chubu University.

I would like to sincerely acknowledge Prof. T. Mutoh, who has been a major member of Chubu University's ImPACT research since 2016 and Dr K. Niita for his development and guidance on the specification of the PHITS code. Additionally, I am deeply grateful to Riken's Prof H. Sakurai for providing the physical guidance of this research. I also deeply thank Professor T. Yoshida, Dr A. Matsubara and Dr Y. Hasegawa.

We would like to express our gratitude to Professor $\mathrm{K}$. Ito for his great help about concept of muon neutron sources.

[1] M. Shimada et al., "Progress in the ITER Physics Basis, Chapter 1: Overview and Summary", Nucl. Fusion 47, S1 (2007).

[2] H. Sagara, T. Yoshida and M. Saito, Proceedings of ICONE 10, 22753 (2002).

[3] A. Takibayev, M. Saito, V. Artisyuk and H. Sagara, Prog. Nucl. Energy 47, 354 (2005). 
[4] S. Chiba, T. Wakabayashi, Y. Tachi, N. Takaki, A. Terashima, S. Okumura and T. Yoshida, Scientific Reports 7, 13961 (2017).

[5] H. Sagara, T. Yoshida and M. Saito, Proceedings of 10th International Conference on Nuclear Engineering; 2002 April 14-18; Arlington, Virginia, USA: Nuclear Engineering Division 4, 22753 (2002).

[6] Y.B. Zeldovich and S.S. Gerstein, Sov. Phys. Uspekki 3, 593 (1961).

[7] K. Nagamine, Introductory Muon Science (Cambridge University Press, Cambridge, 2003).

[8] Y. Kino and M. Kamimura, Hyperfine Interact 82, 45 (1993).

[9] P. Froelich, A. Flores-Riveros, J. Wallenius and K. Szalewicz, Phys. Lett. A 189, 307 (1994).

[10] A. Iiyoshi, Y. Kino, M. Sato, Y. Tanahashi, N. Yamamoto,
S. Nakatani, T. Yamashita, M. Tendler and O. Motojima, AIP Conf. Proc. 2179, 020010 (2019).

[11] M. Sato, Y. Kino, Y. Tanahashi, N. Yamamoto, H. Takano, T. Mutoh, A. Fujita, A. Iiyoshi and A. Matsubara, Proc. of 2018 Fall Meeting Atomic Society of Japan, Okayama, Japan, 2N03 (2018).

[12] T. Sato, Y. Iwamoto, S. Hashimoto, T. Ogawa, T. Furuta, S. Abe, T. Kai, Pi-En. Tsai, N. Matsuda, H. Iwase, N. Shigyo, L. Sihver and K. Niita, J. Nucl. Sci. Technol. 55, 684 (2018).

[13] K. Shibata, O. Iwamoto, T. Nakagawa, N. Iwamoto, A. Ichihara, S. Kunieda, S. Chiba, K. Furutaka, N. Otuka, T. Ohsawa, T. Murata, H. Matsunobu, A. Zukeran, S. Kamada and J. Katakura, J. Nucl. Sci. Technol. 48, 1 (2011).

[14] M.R. Gilbert and J-Ch. Sublet, Nucl. Fusion 51, 043005 (2011). 